Linguistique, littérature, didactique

\title{
Une étude de cas : le lexique d'une classe de cours préparatoire en ZEP
}

\section{Marceline Laparra}

\section{(2) OpenEdition}

\section{Journals}

\section{Édition électronique}

URL : http://journals.openedition.org/pratiques/3483

DOI : $10.4000 /$ pratiques.3483

ISSN : 2425-2042

\section{Éditeur}

Centre de recherche sur les médiations (CREM)

\section{Édition imprimée}

Date de publication : 15 décembre 2012

Pagination : 257-278

\section{Référence électronique}

Marceline Laparra, «Une étude de cas : le lexique d'une classe de cours préparatoire en ZEP »,

Pratiques [En ligne], 155-156 | 2012, mis en ligne le 18 décembre 2017, consulté le 01 mai 2019. URL http://journals.openedition.org/pratiques/3483; DOI : 10.4000/pratiques.3483 


\title{
Une étude de cas : le lexique d'une classe de cours préparatoire en ZEP
}

\author{
Marceline Laparra \\ Université de Lorraine, CREM
}

S'il y a bien un consensus chez tous les acteurs de l'école (parents, enseignants, ministère et ses représentants sur le terrain, chercheurs) c'est celui qui concerne le rôle affirmé comme décisif de la compétence lexicale des élèves dans la réussite des premiers apprentissages scolaires. L'existence d'un déficit en la matière qui existerait chez nombre d'élèves issus de milieu populaire est régulièrement invoqué aussi bien dans les débats destinés au grand public que chez les spécialistes (A. Bentolila 1996) pour expliquer l'échec scolaire se manifestant dès la grande section de maternelle et concernant essentiellement l'apprentissage de la lecture.

On peut donc s'attendre à ce que la très grande majorité des enseignants accorde tout au long du cycle 2 de l'école primaire (dernière année de la maternelle et deux premières années de l'école élémentaire) une très forte place au développement du lexique. Mais quelle réalité recouvre cette notion ? Si on peut avoir une idée assez précise des activités qu'ils mettent alors en œuvre, on sait peu de choses sur les choix qu'ils effectuent et sur quoi ils s'appuient alors pour le faire. Ils opèrent au fil de la journée constamment des sélections lexicales pour chacune des tâches, que celles-ci aient explicitement ou non des visées lexicales.

\section{Difficultés rencontrées lors de la sélection lexicale}

Ils doivent tenir compte de deux impératifs qui peuvent sembler contradictoires : la réussite des activités proposées exige que soient sollicités au maximum des termes déjà connus des élèves, qui ont été souvent acquis lors des échanges quotidiens, scolaires et extra scolaires et que soient introduits simultanément de nombreux termes inconnus d'eux.

Ils se trouvent alors devant une série de difficultés.

Comment déterminer ce qui est connu et ce qui est nouveau à la fois pour chaque élève pris individuellement et pour l'ensemble qu'ils forment, l'hétérogénéité existant entre les élèves semblant particulièrement forte en ce domaine? 
Ce qui est connu l'est-il à la fois en compréhension et en production ? L'évanescence des échanges oraux leur interdit de s'en faire une représentation autrement qu'intuitivement. À la différence de ce qui se passe pour la syntaxe (François et alii, 1972), ils ne peuvent se référer à des descriptions de ce qu'est censé être le bagage lexical d'un enfant de 5-6 ans à la fois proposées par de chercheurs et validées par l'institution scolaire. Cette dernière se contente en effet de fixer des objectifs généraux, sans fournir aucune aide efficace pour cerner le lexique à étudier. ( $C f$. par exemple M.E.N., 1997 : 34-36). Les enseignants ne peuvent s'appuyer sur aucune liste de vocabulaire : celles qui existent (Ters et alii 1970, Catach 1984) ne valent que pour les apprentissages orthographiques.

Si de telles listes ne sont pas disponibles, c'est parce qu'il n'existe pas de critères objectif permettant de les constituer. Le seul critère qu'il est possible de retenir, celui de fréquence, ne permet que d'isoler le vocabulaire dit fondamental (Gougenheim et alii 1964), qui est celui utilisé dans les situations de communications ordinaires et qui est précisément celui qui largement maîtrisé par tous les élèves, au moins de manière passive, à l'âge de 6-7 ans.

Certains enseignants, pour sortir de ces difficultés, pourraient être tentés de s'inscrire dans la tradition scolaire qui dans la première moitié du siècle dernier organisait le lexique au $\mathrm{CP}$ par thèmes, en proposant pour chaque thème étudié durant une semaine une vingtaine de mots comprenant des noms, des adjectifs et des verbes pour répondre aux besoins des très nombreux élèves qui parlaient alors en famille des dialectes du français ou des dialectes d'autres langues minoritaires ${ }^{(1)}$.

Mais en ce cas se poserait une nouvelle difficulté : les thèmes sélectionnés par les manuels de l'époque renvoient majoritairement à un monde rural peu familier des élèves contemporains. Il faudrait les remplacer par d'autres mieux adaptés aux besoins d'élèves très majoritairement francophones, qu'ils soient ou non locuteurs natifs du français. Sur quoi se fonder pour les choisir et quels termes retenir pour chaque thème? L'absence d'une réflexion collective sur ce problème explique sans doute qu'on ne retrouve plus dans les classes que de manière affaiblie cette logique thématique. Existe-t-il des critères repérables qui fondent les choix lexicaux des enseignants?

\section{Critères de sélection lexicale}

Pour ne pas en rester au stade des questions sans réponse, il m'a semblé utile d'analyser le lexique d'une classe particulière. Les conclusions auxquelles j'arrive au terme d'une telle étude de cas ne sont évidemment pas généralisables sans de grandes précautions (Passeron \& Revel 2005). Les informations qu'elles apportent sur la taille et la composition du lexique qui sont propres à cette classe sont moins importantes que celles concernant le mode de sélection des termes. Les facteurs qui entrent en jeu pour déterminer les choix lexicaux influencent en effet la nature du lexique. Le résultat - le lexique - permet d'analyser son processus d'élaboration. Apparaissent un certain nombre de phénomènes qui eux peuvent être considérés comme ayant une portée plus générale, permettant de faire quelques suggestions didactiques.

(1) Lelu et alii 1935. Une telle logique prévaut encore souvent en FLE. On peut repérer dans le lexique de cette classe la présence de certains sous-ensembles mais qui ne sont pas présentés en tant que tels aux élèves. À titre d'exemple, on trouvera en annexe IV regroupés des noms de parties du corps humain ou animal. 


\subsection{Le corpus utilisé}

Dans le cadre d'un travail entrepris au sein du réseau RESEIDA ${ }^{(2)}$, Claire Margolinas et moi-même avons recueilli en 2005-2006, dans une Grande Section d'école maternelle et un Cours Préparatoire (CP) de deux écoles d'une petite ville ouvrière du Puyde-Dôme scolarisant la même cohorte d'élèves, tous les travaux écrits collectifs ou individuels produits dans ces deux classes. Nous disposons en outre d'une cinquantaine de transcriptions d'enregistrements vidéo de séances de 30 à 45 minutes chacune, portant essentiellement sur le français et les mathématiques. Au CP, le manuel de mathématiques utilisé est familièrement appelé par les élèves « le Picbille» (Brissiaud 2002) et l'apprentissage de la lecture s'effectue sans le recours à une méthode, à partir des textes de plusieurs albums de littérature de jeunesse, selon la méthodologie préconisée par des didacticiens comme C. Tauveron ou A. Bentolila ( $c f$. par exemple S. Plane 2004).

Ce corpus m'a permis, pour le $\mathrm{CP}$, de dresser la liste de tous les termes présents au moins une fois à l'écrit ( $c f$. Annexes), non compris les morphèmes-mots (prédéterminants, pronoms, prépositions, conjonctions, adjectifs indéfinis, adverbes du type aussi, beaucoup, mieux, même, trop)

Ce lexique écrit ainsi obtenu comprend un peu plus de 1.500 termes dont à peu près 360 verbes, 150 adjectifs-participes et 950 noms, le reste étant des lexies verbales et des locutions adverbiales ${ }^{(3)}$.

Ces 1.500 termes ne représentent assurément pas la totalité du lexique utilisé dans cette classe ; nombre de mots apparus dans les interactions verbales n'ayant pas nécessairement laissé une trace écrite : ainsi le terme diagonale est-il présent dans les enregistrements oraux et absent de la liste écrite. Certains mots fonctionnent comme des couples; dans le corpus l'un peut être représenté et l'autre non : neiger mais pas pleuvoir, tante mais pas oncle, etc. Manquent aussi des mots à très haute fréquence, connus obligatoirement par tous les élèves par exemple : avancer, battre, boulanger, conduire, encre, épicier, éponge, essuyer, gai, huile, incendie, payer, sable, vif, vin etc.

On peut penser que hors les mots-morphèmes, le lexique employé durant cette année de CP tourne sans doute autour de 2000 termes. Les interactions orales conservées dans les transcriptions et les réponses individuelles fournies à l'écrit me permettent d'estimer que plus de $80 \%$ de ce lexique fait partie à la fin du CP du vocabulaire passif de quasiment tous les élèves, même ceux qui sont les plus en difficulté.

\section{2. Étendue du lexique}

On peut d'autre part affirmer que cette classe de $\mathrm{CP}$ a un lexique peu étendu. Si on procédait à un tel décompte dans d'autres classes, on obtiendrait vraisemblablement des résultats supérieurs, ceci pour deux raisons différentes :

a) La classe observée qui est située en ZEP, scolarise presque exclusivement des élèves de milieu populaire, très défavorisés sur le plan économique et culturel. Comme ses élèves sont censés avoir un déficit linguistique important, l'enseignante essaye

(2) Le réseau RESEIDA regroupe sous l'égide de l'équipe ESCOL de Paris 8 des chercheurs de plusieurs laboratoires autour des processus de différenciation scolaire.

(3) Certains mots sont recensés dans une catégorie grammaticale mais ils auraient pu l'être dans une autre. 
que la langue de l'école soit la plus proche possible ce qu'elle suppose être la langue de ses élèves, pour favoriser au maximum leur réussite. Elle affiche de ce fait des objectifs très modestes au plan linguistique, même si la langue qu'elle utilise ellemême se démarque nettement de celle de ses élèves, par sa diction, sa syntaxe plus proche de l'écrit et son souci constant d'éviter tous les tours trop familiers. Elle reproduit l'attitude de nombreux maîtres placés dans la même situation qu'elle, les enseignant travaillant en ZEP et faisant le choix inverse d'introduire en classe un vocabulaire varié et abondant étant sans doute très minoritaires.

b) La deuxième raison tient au matériel utilisé pour l'apprentissage de la lecture : les histoires racontés dans les albums choisis sont courtes et répétitives; elles mettent en scène essentiellement des animaux familiers. Le lexique qui y est employé est majoritairement à haute fréquence et ne présente qu'une cinquantaine de termes difficiles à comprendre. Les fiches grâces auxquelles s'effectue l'essentiel du travail phonographique ne présentent pas une grande dispersion lexicale, à la différence de ce qui se passe dans beaucoup des méthodes de lectures utilisées de nos jours, les mêmes mots étant réutilisés pour étudier des relations phonographiques différentes et chaque relation phonographique étant illustrée par un nombre restreint de termes.

A titre de comparaison, une méthode comme Un monde à lire qui utilise elle aussi des albums comme supports mais procède à une analyse du code plus détaillée introduit en deux leçons, celles portant sur le [1] et le [m] 6 mots qu'on ne retrouve pas dans le lexique de mon étude : amitié, campagne, île, litre, minute, salive ${ }^{(4)}$.

Plusieurs facteurs expliquent la composition de cet ensemble lexical.

\subsection{Poids déterminant des objectifs phonographiques et orthographiques}

Les activités sur l'unité-mot sont largement dominantes, quand on les compare à celles effectuées sur des unités de rang supérieur comme le syntagme, la phrase ou le texte. Parmi elles, celles qui ont une visée phonographique, et à un moindre degré orthographique, sont largement majoritaires.

Le mot y est alors moins envisagé dans sa dimension sémantique ou morpho-syntaxique que dans sa dimension phonologique : il est le plus souvent sélectionné parce qu'il présente un phonème (ou un groupe de phonèmes) placé dans une position déterminée (initiale, médiane, ou finale) ou parce qu'il est fait d'une combinaison de syllabes simples.

Le travail phonologique, quand il est effectué essentiellement à l'aide de fiches individuelles comme c'est le cas ici, suppose que l'élève qui n'est encore qu'un apprenti lecteur puisse trouver seul le signifiant oral du terme sur lequel il exerce sa réflexion. Pour ce faire, il a à sa disposition soit un signifiant écrit accompagné d'une illustration représentant un objet du monde supposé être le référent et donc le sens du mot en question, soit uniquement une illustration.

Cela entraîne une sur-représentation de la classe des noms, et dans celle-ci de la classe des noms concrets et une sous-représentation des autres classes de mots qui sont malaisés à illustrer. Les mots doivent en outre être présents dans le vocabulaire passif des élèves. La majorité des noms de ce lexique sont donc soit des noms d'animaux ou de plantes soit des mots correspondants à des objets présents dans l'environnement quotidien des élèves et donc des mots déjà connus et souvent présents dans

(4) $C f$. aussi les vingt tableaux de mots contenant un phonème du français, pp. 317-335 du guide pédagogique de la méthode d'apprentissage de la lecture Rue des Contes. 
les fiches utilisées en GS de maternelle. En outre un très grand nombre d'entre eux se prêtent à une décomposition en syllabes simples (domino, moulin, limace, lilas, etc.).

L'ensemble ainsi obtenu peut sembler aller de soi pour l'enseignant pour deux raisons : il renvoie à la représentation commune du lexique comme une nomenclature du monde et il ne présente que des différences au plan quantitatif avec le lexique de toutes les méthodes de lecture produites depuis plus d'un siècle où on lave toujours la salade et où l'amie de maman est malade! Les seules différences tiennent dans le fait que anciennement c'est l'âne qui mâche des coquelicots alors que de nos jours c'est plutôt le crocodile qui se repose près de la mare.

Les mots abstraits, largement sous-représentés, sont le plus souvent ceux qui se décomposent en syllabes de base comme vérité, visite, nature ou qui fournissent un exemple de graphie complexe comme malheur ou sommeil.

Ces phénomènes sont présents dans toutes les classes. Ce qui varie d'une classe à l'autre dépend essentiellement de l'importance plus ou moins grande donnée au travail sur la combinatoire syllabique. Selon qu'elle est très pratiquée ou non, l'étendue du lexique varie mais ce qui est constant, c'est le fait qu'y dominent les termes concrets. D'une classe à une autre ils peuvent être plus ou moins familiers pour les élèves.

La sous-représentation des autres classes de mots est inévitable et elle se retrouve dans toutes les classes.

Les adjectifs-participes sont très peu nombreux et ils forment un stock dépourvu de toute organisation. La seule série repérable est celle des adjectifs de couleur.

La classe des verbes est restreinte à ceux d'entre eux qui peuvent être illustrés par une vignette représentant un petit personnage ou un animal en train d'effectuer une action, ou à l'aide d'un code visuel (une oreille, un œil...). L'élève travaille alors essentiellement sur des verbes à haute fréquence, faisant le plus souvent déjà partie de son vocabulaire actif, verbes qui sont aussi présents dans les albums supports : boire, courir, dormir, jouer, manger, plonger, sauter... Les autres verbes présents dans le corpus (verbes de perception, de sentiment et de mouvement) sont introduits dans les albums. Leur nombre peut varier d'une classe à une autre, selon que la langue des albums est plus ou moins riche.

\subsection{Poids de la langue des albums}

Dans la classe observée, le vocabulaire des 6 albums (Viens jouer avec moi petite souris, La chenille qui fait des trous, L'Intrus, Jean-Loup, Les trois brigands, L'Afrique de Zigomar) n'assure qu'un très faible rééquilibrage, au détriment des mots concrets ayant une référence facilement illustrable. Ceci s'explique parce que les univers de fiction qui sont les leurs sont très proches de ceux des contes : les lieux stéréotypés ne font l'objet d'aucune description (ce sont des montagnes, des cavernes, des châteaux) et les personnages qui sont ou des animaux anthropomorphisés (rat, souris, chenille, éléphant, cigogne...) ou des figures enfantines réduites à leur seul nom, effectuent des actions elles aussi stéréotypées (courir, s'envoler, faire peur, écraser, attraper...).

Quelques séries organisées apparaissent cependant :

- Des adjectifs servant à qualifier un événement ou le comportement et les sentiments d'un personnage : affreux, audacieux, courageux, étranger, formidable, 
furieux, imposant, inquiet, insolent, magnifique, minuscule, obstiné, terrible... - Des noms servant de désignateurs de personnage, dans les chaines anaphoriques : compère, ennemi, explorateur, indigène, intrus, invité, mangeur de, membre de la tribu, otage, passager, promeneur, visiteur, voyageur...

- Des verbes introducteurs de dialogue. Les deux derniers albums en présentent une grande variété : conseiller, consentir, constater, se défendre, s'écrier, s'esclaffer, s'exclamer, faire, hurler, implorer, s'indigner, s'inquiéter, insister, s'insurger, s'interroger, interrompre, s'obstiner, protester, se réjouir, rétorquer, ricaner, sangloter, soupirer, suggérer, supplier...

\subsection{Poids des disciplines autres que le français et les mathématiques}

On pourrait penser qu'un apprentissage systématique d'autres champs lexicaux est effectué lors d'autres activités que celles qui sont explicitement tournées vers les apprentissages en lecture-écriture.

Mais comme dans beaucoup d'autres classes de ZEP, l'enseignante accorde une place dominante au français et aux mathématiques (le fichier de $J^{\prime}$ apprends les maths avec Picbille est systématiquement utilisé et son étude est conduite jusqu'à son terme). Le fait de les considérer comme des disciplines fondamentales dont la mâ̂trise est particulièrement importante pour des élèves de milieu défavorisé réduit les autres disciplines à la portion congrue. L'organisation de la classe en groupes de niveaux deux demi-journées par semaine pour le français et la résolution de problème accentue encore ce phénomène, comme le faisait déjà pour la GS le dispositif d'aide personnalisée, centré sur la remédiation phonographique.

Les séquences qui relèvent des autres disciplines sont regroupées sous l'entrée «éveil » et sont quantitativement peu nombreuses (12 en tout sur l'année), occupant chacune plusieurs séances. Les thèmes traités sont quelque peu disparates et s'inscrivent le plus souvent dans une longue tradition scolaire : les cinq sens - le plan de classe - l'évaporation de l'eau - les plantes du potager - les articulations du corps - la germination du haricot - le printemps - la visite de la ferme et d'un élevage de cerfs.

Cinq séquences sont consacrées à l'étude des aliments : la provenance des aliments - le rôle des nutriments - l'équilibre nutritionnel- les groupes alimentaires. Si elles s'inscrivent elles aussi dans la tradition hygiéniste de l'école, elles répondent à des injonctions récentes du ministère concernant la lutte contre l'obésité.

Le vocabulaire introduit appartient le plus souvent à des langues de spécialité (botanique, biologique) et les élèves rencontrent alors des termes savants qu'ils n'auront que rarement l'occasion de lire ultérieurement et encore moins de réemployer comme cotylédon, plantule, nutriment, féculent, insectivore, granivore, noms des bois du cerf...

\subsection{Poids de l'enseignement grammatical}

Dans cette classe, l'enseignante n'accorde qu'une place restreinte à l'apprentissage de connaissances morpho-syntaxiques ou métalinguistiques. Une poignée de fiches proposent des activités ayant cette dimension, telles celle où les élèves doivent engendrer le féminin de quelques adjectifs qui sont essentiellement des adjectifs de couleur.

Elle est ainsi très loin de la manière dont l'école primaire à ses débuts enseignait une grande partie du lexique, en s'appuyant sur d'autres outils que le manuel d'ap- 
prentissage de la lecture. Il existait en effet dès le CP des manuels de langue qui pour chaque entrée grammaticale fournissaient des listes de termes regroupées par thèmes, combinant alors une logique grammaticale à une logique thématique : ainsi pour les noms de personne, une trentaine de noms de métier, pour les noms d'animaux des noms d'animaux domestiques et des noms d'animaux sauvages, pour les noms de choses des noms d'outils et d'arbustes et de fruits, pour les adjectifs qualificatifs des adjectifs « marquant la couleur », " marquant la forme et la grandeur », " marquant les qualités ou les défauts ${ }^{(5)}$. Ces manuels de langue, comme les manuels de vocabulaire évoqués plus haut, ont pratiquement disparu au CP. Ce sont les manuels de lecture qui prennent en charge une partie de cet enseignement, en intégrant dans le travail sur la compréhension la reconnaissance de certains morphèmes grammaticaux. L'absence dans cette classe de tout manuel d'apprentissage de la lecture explique que les objectifs grammaticaux ne jouent pratiquement pas dans la sélection du lexique.

Pour résumer, on voit que les choix lexicaux effectuées par l'enseignante de cette classe sont majoritairement dominés par deux nécessités :

- Celle d'utiliser au maximum des termes appartenant au vocabulaire connu des élèves (et à leur monde de référence).

- Celle de pouvoir représenter le référent des termes qui servent de support au travail phonographique.

Les contraintes induites par la volonté d'enseigner aux élèves d'autres connaissances de type lexical (grammaticales ou purement orthographiques) ne jouent qu'à la marge ici, alors que dans d'autres classes, elles peuvent s'exercer de manière plus importante et donc modifier quelque peu l'étendue du lexique mais pas sa constitution.

\section{Problèmes soulevés par un lexique ainsi constitué}

Si on ne considère que l'étendue du lexique, on peut en un premier temps penser que l'enseignante respecte parfaitement les objectifs fixés par les Instructions officielles : les élèves disposent d'un vocabulaire qui dépasse largement celui que l'on leur prête souvent quand il s'agit d'élèves de milieu défavorisé. Cette étude de cas dément l'idée souvent exprimée dans des débats destinés au grand public, selon lesquels les élèves qui seraient en difficulté lors de l'apprentissage de la lecture seraient en échec puisqu'ils ne disposeraient que d'un stock de mots très limités (800 à 1000 mots, parfois moins).

Le lexique utilisé ne leur pose que peu de problème de compréhension. Il leur permet de mettre en œuvre ce que les Instructions officielles appellent « le langage d'évocation » et il est sans doute suffisant pour effectuer sur les objets du monde les activités cognitives que l'on attend d'eux.

Les interactions orales lors des activités de compréhension des textes montrent que ce ne sont pas des problèmes sémantiques qui sont très majoritairement à la source de l'échec en lecture. Cela est d'autant plus intéressant à souligner que pour toutes les raisons exposées plus haut cette classe use d'un lexique dont l'étendue est sans doute inférieure à celle de bien d'autres classes et qu'on peut supposer que peu de classes, en milieu défavorisé, utilisent un vocabulaire moins varié.

(5) Cf. par exemple Maquet et alii. 
Les termes qu'ils soient connus ou inconnus ne posent au $\mathrm{CP}$ que peu de problèmes de polysémie, alors que celle-ci devient dès le cours élémentaire un obstacle majeur à la compréhension des énoncés ; les termes déjà connus sont souvent polysémiques, mais les élèves en maîtrisent les sens qui sont les leurs dans les situations familières (la maison est le lieu où eux et leur famille habitent et elle est un type d'habitation particulière). Les termes nouveaux sont le plus souvent introduits avec une seule acception.

Néanmoins, si la situation peut sembler satisfaisante si on s'en tient à ce qui se passe au CP, il n'en va pas de même si on pense à la suite de la scolarité. Plusieurs problèmes apparaissent alors.

\subsection{Rapport entre ce qui est connu et ce qui est nouveau}

Plus de $80 \%$ des termes qui constituent ce lexique sont déjà connus des élèves. Ils étaient déjà largement employés en GS. Ils couvrent, je l'ai dit, les besoins de communication des élèves et les besoins engendrés par le travail effectué en classe. Mais couvrent-ils les besoins qui seront les leurs dès l'entrée en cours élémentaire?

S'il est normalement souhaitable que la réflexion phonographique et les activités de production et de compréhension des textes soient effectuées, comme c'est le cas ici, majoritairement à l'aide d'un lexique appartenant déjà au vocabulaire passif des élèves, ne serait-il pas souhaitable, sans pour autant leur demander d'apprendre des listes de mots, à l'instar de ce qui se fait parfois en langue vivante, de leur proposer des activités ayant pour unique objectif d'introduire en plus grand nombre des termes nouveaux, surtout dans la deuxième partie de l'année?

\subsection{Part faite au vocabulaire renvoyant à un univers enfantin}

Une partie non négligeable de ce lexique (certains des noms d'animaux ou d'objets dont la charge affective est grande) verront leur fréquence diminuer rapidement au cours de la scolarité. Là encore, même si le vocabulaire doit être sélectionné en tenant compte des centres d'intérêt d'élèves âgés de six ans, il n'est pas nécessaire de privilégier parmi eux ceux qui ne seront plus ceux d'élèves plus âgés, ainsi qu'on le fait trop souvent.

\subsection{Nature du travail effectué sur ou à l'aide de ce lexique}

Si on envisage le lexique de cette classe d'un point de vue plus qualitatif les constats plutôt positifs qui pouvaient être dressés jusque là se nuancent fortement : le travail qui est effectué sur lui conduit à s'interroger sur le fait que les élèves puissent en avoir dans les classes suivantes un usage actif :

On l'a vu précédemment, les termes sur lesquels s'exerce l'activité phonographique, plus rarement orthographique, ne sont pas l'objet d'un traitement en discours, mais en langue. Très souvent, même si l'enseignante vérifie toujours quand elle explique la tâche à accomplir que les élèves sont capables de reconnaître l'objet du monde que le mot sert à désigner, au cours de l'activité elle-même la signification du terme passe au second plan.

Certains termes ne sont envisagés que comme une combinatoire de syllabes simples dont l'élève est censé pouvoir oraliser facilement le signifiant, puisqu'il connait les dites syllabes ; il ne voit alors pas leur sens être précisé, ce qui est malheureuse- 
ment souvent le cas, ainsi que je l'ai déjà dit, pour un certain nombre de mots abstraits comme nature, parole, vérité, visite. Leur présence dans les listes figurant en annexe ne veut pas dire qu'ils aient réellement un sens pour la grande majorité des élèves.

De la même manière certains termes ne sont que des modèles à reproduire dans les exercices de copie, ou ne sont qu'un assemblage de lettres à retrouver parmi une collection d'assemblages lui ressemblant (par exemple maman parmi main, manier, manche, nommer) ${ }^{(6)}$.

A l'inverse, les termes nouveaux apparaissant dans les albums voient leur sens être expliqué au fil des interactions orales, mais ils ne sont que rarement ensuite l'objet d'un travail définitoire hors contexte, ce qui conduite à douter que tous les élèves soient capables d'en donner un sens en langue. Ce n'est qu'en fin d'année que les élèves ont à fournir des réponses à des « devinettes » :

« [...] Elles vous permettent d'entrer : les ...

Dans les champs, il doit faire peur aux oiseaux : un...

La marraine de Cendrillon en a pris une grosse pour la transformer en carrosse. Une... [...]»

Il s'agit alors moins de trouver le mot qui correspond à une définition que de trouver à quel objet du monde renvoie ce qui est écrit. Ce jeu de devinette figure sur une fiche consacrée aux graphies de [ij], [عj], [aj], [uj], [œj].

L'apparition successive des termes ne répond pas à une logique interne. C'est à la fois l'ordre dans lequel sont travaillés les phonèmes et la place des mots dans les textes qui font que domino est rencontré dès les premiers jours et maillot en fin d'année. Croquer est présent dans un album travaillé au premier semestre, décoller dans le dernier album.

D'autre part, on l'a vu, certains mots n'ont pas toujours leurs antonymes : gros est utilisé, mais pas maigre.

Ces phénomènes interdisent pratiquement à l'enseignant de faire prendre conscience à ses élèves qu'il existe entre les mots des relations sémantiques et des relations morphologiques.

\section{Relations sémantiques :}

Il est normal au CP de ne pas introduire un métalangage servant à les décrire. Mais on peut néanmoins commencer à faire trouver aux élèves quel est le lien entre garçon, fille, homme, femme, enfant, à leur montrer que le contraire de rapide est lent, que les tigres et les lions sont des félins, que l'hirondelle et le merle sont des oiseaux qui sont des animaux. L'étendue du lexique le permet parfaitement (tous les termes cités en font partie), mais pas la manière dont il est constitué au fil des jours.

\section{Relations morphologiques :}

Pour les mêmes raisons, l'enseignante ne peut que rarement procéder à des rapprochements entre des termes comme plonger et plongeon, se baigner et baignade, place et emplacement, toit et toiture. On ne trouve guère comme activités de ce type, toutes faites en fin d'année, que celle déjà évoquée concernant le féminin de certains adjectifs et celles qui permettent de dériver un nom d'un verbe.

(6) C'est la raison pour laquelle manier, manche et nommer ne figurent pas sur les listes présentées en annexe. 
« Retrouve ce qu'ils font (exemple : le boxeur $\rightarrow$ il boxe)

Le chasseur $\rightarrow$ il...

Le voleur $\rightarrow i l \ldots$

Le danseur $\rightarrow i l \ldots$

Le tricheur $\rightarrow$ il...

Le nageur $\rightarrow i l \ldots$

Retrouve ce qu'ils sont (ex : il chante $\rightarrow$ le chanteur)

Il ment $\rightarrow$ le...

Il vend $\rightarrow l e \ldots$

Il joue $\rightarrow$ le...

Il invente $\rightarrow l^{\prime} \ldots$

Il coiffe $\rightarrow$ le... »

\subsection{Place accordée aux termes n'ayant pas pour référence un objet du monde ou n'ayant pas une référence discrète}

Les mots abstraits, les collectifs comme famille ou les massifs comme liquide sont peu nombreux et ils sont souvent en lien avec le travail effectué en classe (vocabulaire des consignes, lexique spécialisé du français et des mathématiques, termes renvoyant à la gestion du temps et de l'espace, $c f$. annexe).

La faible étendue de ce sous-ensemble lexical (en annexe) et son caractère disparate font problème si on pense à ce que seront dès le cours élémentaire les besoins en compréhension et en production des élèves. Comment justifier l'absence de raison, alors qu'indiscrétion est présent?

Mais surtout les deux listes fournies en annexe peuvent faire illusion : s'y trouvent plusieurs termes qui n'apparaissent que dans des lexies verbales ou des locutions figées : chance, chasse, compte, droit, doute, façon, faim, énergie, envie, essai, justesse, faim, fuite, horreur, impression, intention, place, peine, peur, réalité, recherche, secours, soif, suite, temps, volonté.

Leur utilisation dans des syntagmes plus ou moins figés ne les rend pas automatiquement disponibles comme noms capables d'entrer dans des combinatoires syntaxiques nouvelles. Pour ce faire, il faudrait que soit procédé à un travail spécifique du type : « il a l'impression de... Il ne s'est pas trompé son impression était juste.»

« Il a pris la place de... et cette place n'était pas la sienne. » (Laparra 2005)

Si on soustrait de ces deux listes ces termes, ainsi que ceux qui n'ont été l'objet que d'un travail sur les syllabes qui les compose, on s'aperçoit que ce qui reste est réduite à la portion congrue.

\subsection{Réemploi des termes dans les différents types d'activité}

La majorité des termes de cette liste sont réemployés à de nombreuses reprises par les élèves :

- Les termes relevant du travail scolaire sont utilisés quotidiennement.

- Un même terme, on l'a vu, peut être utilisé pour illustrer des relations phonographiques différentes.

- Les termes introduits par les albums sont l'objet d'un travail intensif durant les 
cinq ou six semaines où l'album sert de support aux apprentissages : copie, dicté, questions de compréhension, exercices syntaxiques, suite ou résumé de l'histoire, toutes ces activités font qu'un même terme peut être réemployé durant près d'un mois, parfois plus d'une vingtaine de fois.

A première vue, de telles pratiques, si elles limitent, ainsi qu'on l'a vu, l'étendue du lexique, permettent la mémorisation des termes nouveaux et assure leur entrée dans le vocabulaire passif de tous les élèves.

Mais s'il y a bien réemploi des termes nouveaux, il ne s'accompagne pas d'un processus de décontextualisation : ces termes sont toujours utilisés dans le même contexte référentiel. On observe bien en situation des connaissances lexicales relativement importantes chez les élèves, mais celles-ci ne peuvent devenir des connaissances utiles en discours, encore moins des savoirs en langue (Laparra \& Margolinas 2010), faute d'un réemploi effectué dans des contextes référentiels différents et après que ce soit écoulé un laps de temps suffisant après leurs premières réutilisations.

\section{Conclusion}

Le lexique de cette classe présente certes de nombreuses particularités, liées notamment à l'appartenance socioculturelle des élèves et à la méthodologie suivie dans l'apprentissage de la lecture mais il autorise au-delà de ces spécificités, à procéder à certaines généralisations.

Les élèves sortent de la GS avec un vocabulaire passif important sur lequel le CP s'appuie très largement. Y dominent des noms d'animaux, de personnes, des noms de choses renvoyant à la vie quotidienne et des verbes d'action. Ce lexique assure la compréhension de ce qui est dit et écrit dans la classe, et la partie dont les élèves ont un usage actif est suffisante pour qu'ils puissent comprendre et produire toutes les verbalisations qu'impliquent les situations d'apprentissage auxquels ils sont confrontés. L'échec que rencontrent certains d'entre eux n'a donc pas sa source dans un déficit lexical.

Un lexique nouveau est apporté tout au long de l'année, mais si son étendue est variable d'une classe à une autre, il présente toujours un caractère disparate, peu organisé. Les élèves en ont une connaissance fortement dépendante des situations dans lesquelles ils sont utilisés. La part faite au lexique abstrait, ou qui ne permet pas de référer directement au monde est faible et il n'est pas sélectionné en fonction des besoins qui seront ceux des élèves en cours élémentaire.

Le travail réflexif effectué sur le vocabulaire est très majoritairement phonographique, et dans une part plus ou moins importante selon les classes, orthographique.

Ces phénomènes sont la résultante de plusieurs contraintes qui ont été décrites cidessus et dont la hiérarchie peut ne pas être la même dans toutes les classes. Elles s'exercent sur les enseignants de façon plus ou moins consciente, en fonction de la manière dont ils conçoivent les activités proposés aux élèves. Il serait illusoire de penser que l'on puisse agir directement ou indirectement sur des processus aussi complexes.

Si l'on veut fournir dès le CP à tous les élèves un lexique actif leur permettant de réussir dans les classes suivantes, il faut se garder d'essayer de modifier le lexique tel qu'il apparaît dans cette étude de cas ou celui qui apparaîtrait dans d'autres études de cas, mais il convient plutôt que l'institution scolaire demande aux enseignants de 
mettre en place très régulièrement des activités ayant pour unique objet de construire un ensemble raisonné ${ }^{(7)}$ au plan sémantique et morphosyntaxique de termes nouveaux à dominante abstraite dont la liste doit être fournie aux enseignants, ensemble que seules les familles de milieux favorisés se chargent aujourd'hui de faire acquérir : à titre d'exemple en conclusion on peut se risquer à esquisser trois séries :

- Avoir raison, une raison, la raison, raisonner, le raisonnement

- Changer, un changement/échanger, un échange

- Différent, faire une différence entre/avec, à la différence de, la différence, différencier.

(7) Ensemble qui est à compareravec celui qu'une méthode de lecture comme Ribambelle propose dans un fascicule intitulé «mes outils pour écrire, vocabulaire». 


\section{Références}

Bentolila,A. (1996) : «Lecture et maîtrise de la langue », in Regards sur la lecture et ses apprentissages, Observatoire National de la Lecture, M.E.N., 3-15.

CATACH, N. (1984): Les listes orthographiques de base du français (LOB). Les mots les plus fréquents et leurs formes fléchies les plus fréquentes, Paris : Nathan.

François, D., François, F., SAbeau-Jouannet, E., Sourdot, M. (1972) : La syntaxe de l'enfant avant cinq ans, Paris : Larousse.

Gougenheim, G., MichéA, R., Rivenc, P., SAuvageot, A. (1964) : L'élaboration du français fondamental (1 $1^{\text {er }}$ degré), Paris : Didier.

LAPARRA, M. (2005) : "Capacités langagières en production non fictionnelle », in Pratiques, 125-126, 139-156.

LAPARRA, M., MARgolinas, C. (2010) : «Milieu, connaissance, savoir. Des concepts pour l'analyse de situations d'enseignement », in Pratiques, 145-146, 141-160.

M.E.N. (1997) : La maîtrise de la langue à l'école, CNDP Paris, Savoir-Livre.

PASSERON, J.-C., REVEL, J., eds. (2005) : Penser par cas, Paris : Éditions de l'EHESS.

Plane, S. (2004) : «Continuité-rupture : qu'en est-il de l'apprentissage de la lecture sur le terrain?», Table ronde in Actes de l'Observatoire, ONL, M.E.N., 81-108.

Ters, F., MAYER, G., ReICHENBACH, D. $\left(1970^{2}\right)$ : L'échelle Dubois-Buyse d'orthographe usuelle française, Neuchâtel : H. Messeillier.

\section{Manuels}

Baron, L., Condaminas, A. (2006) : Méthode de lecture Rue des Contes CP, Guide du maître, Paris : Magnard.

Bentolila, A., dir. (2012) : Un monde à lire, Paris : Nathan.

Brissiaud, R., Clerc, P., Ouzoulias, A. (2002) : J'apprends les maths avec Picbille, CP, Paris : Retz.

Demeulemeester, J.-P., dir. (2008) : Ribambelle CP, fascicule « Mes outils pour écrire, vocabulaire ». Paris : Hatier.

Lelu, Ad., Kubler, L., Voelzel, L., Vocabulaire CP et cours élémentaire, Paris : Hatier.

Maquet, C., Flot, L., RoY, L., Cours de langue française. Grammaire et exercices, vocabulaire, orthographe, exercices d'élocution, Paris : Hachette.

\section{Albums, littérature de jeunesse}

Boujon, C. (2000) : L'intrus, Paris : L'école des loisirs.

CARLE, C. (1999) : La chenille qui fait des trous, Namur : Mijade.

Corentin, P. (2001) : L'Afrique de Zigomar, Paris : L'école des loisirs.

KRAUS, R. (1989) : Viens jouer avec moi petite souris, Paris : L'école des loisirs.

KringS, A. (1993) : Jean-Loup, Paris : L'école des loisirs.

Ungerer, T. (2001) : Les trois brigands, Paris : L'école des loisirs. 


\section{Annexes}

Sont en italique les mots supposés inconnus. La capitale sert de repère à l'ordre alphabétique.

\begin{tabular}{|c|c|c|c|}
\hline LES VERBES & (se) Boucher & Danser & Embrasser \\
\hline Abandonner & Bouger & Débarrasser & $\left(s^{\prime}\right)$ Émerveiller \\
\hline Abîmer & Boxer & Décevoir & Emmener \\
\hline Accoucher & Briller & Déchirer & Empailler \\
\hline Acheter & & Décider & Emporter \\
\hline Affamer & (se) Cacher & Décoller & Encadrer \\
\hline Affoler & Calculer & Décorer & Enerver \\
\hline Aider & Caresser & Découper & Enfiler \\
\hline Aiguiser & Casser & Défoncer & $\left(s^{\prime}\right)$ Enfoncer \\
\hline Aimer & Changer & Défiler & $\left(\mathrm{s}^{\prime}\right)$ Enfuir \\
\hline Agiter & Chanter & (se) Déguiser & Enregistrer \\
\hline Ajouter & Chasser & (se) Demander & Entendre \\
\hline Alourdir & Chatouiller & Déménager & Entourer \\
\hline Amener & Chercher & Démolir & Entrer \\
\hline (s')Amuser & Choisir & Demeurer & (s')Envoler \\
\hline (s')Apercevoir & Chuchoter & (se) Déplacer & Eplucher \\
\hline Apparaître & Cocher & Déranger & Equilibrer \\
\hline (s')Appeler & (se) Cogner & Déraper & Espérer \\
\hline Apprendre & (se) Coiffer & Dérober & Espionner \\
\hline (s')Apprêter & Coller & Dérouler & Essayer \\
\hline Arracher & Colorier & (se) Désaltérer & (s')Étonner \\
\hline Arranger & (re)Commencer & Descendre & (s')Évanouir \\
\hline (s')Arrêter & Comparer & Désespérer & (s')Évaporer \\
\hline Arriver & Compléter & Désigner & (s')Éveiller \\
\hline Arroser & Comprendre & Dessiner & Évoquer \\
\hline Aspirer & Compter & Détester & Exagérer \\
\hline (s')Asseoir & Confondre & Dévaliser & Examiner \\
\hline (s')Assoupir & (re)Connaître & Devenir & Exploser \\
\hline Attacher & (se) Construire & Dérober & \\
\hline Attaquer & Contaminer & Digérer & Fabriquer \\
\hline Attendre & Contenir & Dire & Fêter \\
\hline Atterrir & Conseiller & (se) Disnuter & Filer \\
\hline Attirer & Convenir & $\begin{array}{l}\text { (se) Disputer } \\
\text { se) Distraire }\end{array}$ & Flairer \\
\hline Attraper & Consoler & Donner & (se) Former \\
\hline Avaler & (re)Copier & Dormir & Frôler \\
\hline Avouer & $\begin{array}{l}\text { Correspondre } \\
\text { Couler }\end{array}$ & (se) Dresser & Fumer \\
\hline Badigeonner & Couper & & Gagner \\
\hline (se) Baigner & Courir & (s')Échanper & Geler \\
\hline (se) Balader & Coûter & Éclabousser & Germer \\
\hline (se) Balancer & Créer & Écraser & Glisser \\
\hline Barrer & (se) Croire & $\begin{array}{l}\text { Ecraser } \\
\text { Écrire }\end{array}$ & Grandir \\
\hline Bâtir & Croiser & Effacer & Gratter \\
\hline Blesser & Croquer & Égratigner & Guérir \\
\hline Boire & Cuire & Élever & \\
\hline
\end{tabular}




\begin{tabular}{|c|c|c|c|}
\hline Habiter & Observer & (se) Regarder & Surveiller \\
\hline Hacher & Obtenir & Regretter & \\
\hline (se) Hérisser & (s')Obstiner & Regrouper & Se Taire \\
\hline \multirow[t]{2}{*}{ Hurler } & (s')Occuper & Rejoindre & Téléphoner \\
\hline & Oter & Relier & (se) Tenir \\
\hline Imaginer & Oublier & Remarquer & Terminer \\
\hline Imiter & Ouvrir & Remercier & Tirer \\
\hline Impressionner & & Remplir & Tisser \\
\hline S'indigner & Parler & Remettre & Tomber \\
\hline Indiquer & Pardonner & Remonter & (se) Tortiller \\
\hline$\left(s^{\prime}\right)$ Infiltrer & Partager & Rencontrer & Toucher \\
\hline$\left(s^{\prime}\right)$ Inquiéter & Partir & (se) Rendre à & Tousser \\
\hline Installer & Se passer & Renoncer & Tracer \\
\hline Insulter & Se passer de & Rentrer & (se) Transformer \\
\hline Irriter & Patiner & Se Renseigner & Travailler \\
\hline \multirow[t]{2}{*}{ Inventer } & (re)Peindre & Repasser & Trembler \\
\hline & Pendre & (se) Repérer & Tricher \\
\hline Jeter & Penser & Répondre & (se) Tromper \\
\hline Jouer & Percer & Se Reposer & (re)Trouver \\
\hline \multirow[t]{2}{*}{ Justifier } & Permettre & Repousser & Tuer \\
\hline & Piquer & Reprendre & \\
\hline Lâcher & Plaire & Reproduire & Utiliser \\
\hline Lancer & Pleurer & Réussir & \\
\hline Laver & (se) Plier & (se) Réveiller & Vendre \\
\hline (se) Lever & Plonger & Rire & Venger \\
\hline Libérer & (se) (re)Poser & Ronronner & Verglacer \\
\hline Loger & Porter & Ruisseler & Vérifier \\
\hline \multirow[t]{2}{*}{ Longer } & (se) Précipiter & & Verser \\
\hline & Préparer & Saigner & Visiter \\
\hline Mâcher & Prévenir & Saisir & Vivre \\
\hline Manger & (se) Promener & Sangloter & Voir \\
\hline Manquer & Proposer & Sauter & Voler 1 \\
\hline (se) Marier & (se) protéger & Sauver & Voler 2 \\
\hline (se) Maquiller & Protester & (se) Sauver & Vomir \\
\hline Se Méfier & & Scandaliser & Voyager \\
\hline Mélanger & (se) quitter & Secouer & \\
\hline Menacer & & Semer & «Auxiliaires $»$ \\
\hline Mentir & Raconter & Sentir & Aller \\
\hline Mesurer & Ranger & Séparer & Devoir \\
\hline Mimer & Rassurer & Serrer & Faire \\
\hline Monter & Rater & (se) Servir & Falloir \\
\hline Se Moquer & (être) ravi & Situer & Finir \\
\hline Se Moucher & Réaliser & Soigner & Laisser \\
\hline Mouiller & Recevoir & Sonner & Mettre \\
\hline \multirow[t]{2}{*}{ Mourir } & (se) Réconcilier & Sortir & Pouvoir \\
\hline & Reconstituer & Soulever & Prendre \\
\hline Nager & Recouvrir & Sourire & Rendre \\
\hline Naître & Recueillir & Suffire & Savoir \\
\hline Neiger & Récupérer & Suivre & Vouloir \\
\hline (se) Nourrir & Réfléchir & Supplier & \\
\hline Numéroter & Refuser & Surligner & \\
\hline
\end{tabular}




\begin{tabular}{|c|c|c|c|}
\hline Verbes & Capital & Inattendu & Principal \\
\hline introducteurs & Charmant & Inquiet & Privé \\
\hline de dialogue & Chaud & Insolent & Prochain \\
\hline Conseiller & Cher & Intéressant & Propre \\
\hline Consentir & Confortable & Inutile & \\
\hline Constater & Content & Involontaire & Rapide \\
\hline Demander & Courageux & & Rêveur \\
\hline (Se) Défendre & Cruel & Jaloux & Riche \\
\hline Dire & & Jaune & Rigolo \\
\hline S'Écrier & Différent & Joli & Rond \\
\hline S'Esclaffer & Difficile & Juste & Rose \\
\hline S'Essouffler & Dodu & & Rouge \\
\hline S'Exclamer & Double & Lent & \\
\hline Faire & Droitier & Lisse & Sage \\
\hline Hurler & Drôle & Long & Sale \\
\hline Implorer & Dur & Lourd & Sec \\
\hline S'Indigner & & & Sérieux \\
\hline S'Inquiéter & Egal & Magique & Seul \\
\hline Insister & Empoté & Magnifique & Sévère \\
\hline S'Insurger & Epais & Malade & Simple \\
\hline S'Interroger & Etonné & Maladroit & Solide \\
\hline S'Interrompre & Etrange & Mâle & Sombre \\
\hline S'Obstiner & & Malheureux & Suivant \\
\hline Protester & Facile & Marrant & Superbe \\
\hline Se Réjouir & Fatigué & Marron & Sûr \\
\hline Rétorquer & Faux & Mauvais & \\
\hline Ricaner & Foncé & Méchant & Terrible \\
\hline Sangloter & Formidable & Muet & Têtu \\
\hline Soupirer & Fort & Migrateur & Timide \\
\hline Suggérer & Français & Mince & Traditionnel \\
\hline \multirow[t]{2}{*}{ Supplier } & Froid & Minuscule & Tranquille \\
\hline & Furieux & Moelleux & Triste \\
\hline Adjectifs & & Moustachu & \\
\hline Adulte & Gaucher & & Végétal \\
\hline Aimable & Géant & Noir & Vert \\
\hline Affreux & Gourmand & Normal & Vide \\
\hline Alimentaire & Gradué & Nouveau & Vieux \\
\hline Ancien & Grand & Numérique & Vilain \\
\hline Animal & Gras & Nutritionnel & Violet \\
\hline Audacieux & Gris & & Vrai \\
\hline Aveugle & Grognon & Obstiné & Voûté \\
\hline & Gros & & \\
\hline Bas & & Pareil & Adverbes en -ment \\
\hline Bavard & Habile & Pauvre & Evidemment \\
\hline Bête & Handicapé & Petit & Gentiment \\
\hline Bizarre & Haut & Plat & Heureusement \\
\hline Blanc & Horrible & Plein & Justement \\
\hline Bleu & & Pointu & Naturellement \\
\hline Blond & Identique & Poli & Précieusement \\
\hline Bon & Impertinent & Possible & Rapidement \\
\hline Brute & Imposant & Précieux & Sagement \\
\hline
\end{tabular}




\begin{tabular}{|c|c|c|c|}
\hline Seulement & Ecureuil & Rat & Eskimo \\
\hline Tellement & Eléphant & Renard & Explorateur \\
\hline Tendrement & Escargot & Renne & \\
\hline Timidement & & Reptile & Femme \\
\hline Tristement & Faon & Requin & Fille \\
\hline Violemment & Fauve & Rhinocéros & Frère \\
\hline \multirow[t]{3}{*}{ Vraiment } & Félin & & \\
\hline & Fourmi & Sanglier & Garçon \\
\hline & & Sauterelle & Gendarme \\
\hline LES NOMS & Girafe & Scorpion & Guide \\
\hline Noms d'animaux & Gorille & Serpent & \\
\hline Abeille & Grenouille & Singe & Habitant \\
\hline Agneau & Guêpe & Souris & Homme \\
\hline \multicolumn{4}{|l|}{ Ane } \\
\hline Animal & Hamster & Taupe & Indigène \\
\hline \multirow[t]{2}{*}{ Araignée } & Hérisson & Tigre & Infirmier \\
\hline & Hibou & Tortue & Intrus \\
\hline Baleine & Hippopotame & & Inventeur \\
\hline Bestiole & Hirondelle & Vache & Invité \\
\hline Bête & & Veau & \\
\hline Biche & Insecte & Ver & Jongleur \\
\hline \multirow[t]{2}{*}{ Bœuf } & & & Joueur \\
\hline & Koala & Zèbre & Jumeau \\
\hline \multicolumn{4}{|l|}{ Caille } \\
\hline Caniche & Lapin & Noms d'homme & Laitier \\
\hline Canard & Libellule & Acrobate & Loubard \\
\hline Canari & Limace & Alpiniste & \\
\hline Cerf & Lion & Ami & Magicien \\
\hline Chameau & Loup & Astronaute & Maître \\
\hline Chat & & & Mamie \\
\hline Chenille & Merle & Berger & Mangeur \\
\hline Cheval & Morse & Boucher & Mari \\
\hline Chèvre & Mouche & Boxeur & Marraine \\
\hline Chevreuil & Moucheron & Brigand & Menteur \\
\hline Chevreau & Mouton & & Mère \\
\hline Chien & Moustique & Canaille & Musicien \\
\hline Chouette & Mulet & Chasseur & \\
\hline Cigogne & & Chevalier & Nageur \\
\hline Coccinelle & Oie & Clown & \\
\hline Cochon & Oiseau & Coiffeur & Ogre \\
\hline $\operatorname{Coq}$ & Ours & Compère & Orphelin \\
\hline Corneille & & Copain & Otage \\
\hline Coucou & Panda & Cousin & \\
\hline Crabe & Papillon & & Parent \\
\hline \multirow[t]{2}{*}{ Cygne } & Phoque & Danseur & Passager \\
\hline & Pie & Dentiste & Père \\
\hline Dauphin & Pigeon & & Personnage \\
\hline Dinde & Pingouin & Elève & Pilote \\
\hline Dindon & Poisson & Eleveur & Pirate \\
\hline Dinosaure & Poulain & Enfant & Polichinelle \\
\hline Dragon & Poule & Ennemi & Policier \\
\hline
\end{tabular}




\begin{tabular}{|c|c|c|c|}
\hline Pompier & Bibliothèque & Camion & Couteau \\
\hline \multirow[t]{2}{*}{ Promeneur } & Bijou & Canapé & Couvercle \\
\hline & Bijouterie & Canne & Crayon \\
\hline \multirow[t]{2}{*}{ Reine } & Bille & Car & Crème \\
\hline & Billet & Carrosse & Croissant \\
\hline Sœur & Bise & Carotte & Cuillère \\
\hline \multirow[t]{2}{*}{ Sorcière } & Blé & Carton & Cuisine \\
\hline & Bobine & Casque & \\
\hline Tante & Boîte & Casquette & Dîner \\
\hline \multirow[t]{2}{*}{ Tricheur } & Bol & Case & Dînette \\
\hline & Bonbon & Casserole & Disque \\
\hline Vendeur & Bouclier & Caverne & Domino \\
\hline Visiteur & Bougeoir & Ceinture & Douche \\
\hline Voleur & Bougie & Céréale & Drapeau \\
\hline \multirow[t]{2}{*}{ Voyageur } & Bois & Cerise & \\
\hline & Botte & Chaîne & Eau \\
\hline Noms communs & Bouclier & Chaise & Echelle \\
\hline$\overline{\text { Abreuvoir }}$ & Boulangerie & Chalet & Ecorce \\
\hline Abri & Boulevard & Chambre & Ecurie \\
\hline Aiguille & Bouquet & Champignon & Eglise \\
\hline Algue & Boussole & Chapiteau & Entonnoir \\
\hline Allumette & Bouteille & Chariot & Enveloppe \\
\hline Amande & Boutique & Chaussure & Epée \\
\hline Ambulance & Bouton & Château & Epouvantail \\
\hline Ampoule & Boxe & Chemin & Escalier \\
\hline Ananas & Bracelet & Cheminée & Etage \\
\hline Anchois & Braise & Chêne & Etagère \\
\hline Appareil photo & Branchage & Chocolat & Etiquette \\
\hline Aquarium & Branche & Ciel & Etoile \\
\hline Arbre & Bretelle & Cigarette & Eventail \\
\hline Arme & Brioche & Cinéma & \\
\hline Armoire & Brochette & Ciseau & Fauteuil \\
\hline Arrosoir & Brosse & Citrouille & Féculent \\
\hline Asperge & Brouette & Chou-fleur & Fenêtre \\
\hline Auto & Bruit & Clef & Fenouil \\
\hline Avenue & Bûche & Cloche & Fer \\
\hline Averse & Buisson & Coffre & Ferme \\
\hline \multirow[t]{2}{*}{ Avion } & Bureau & Collier & Fête \\
\hline & Bus & Colline & Feu \\
\hline Bague & & Concombre & Feuille \\
\hline Bain & Cabane & Coquillage & Feutre \\
\hline Balai & Cachette & Corbeille & Fève \\
\hline Balance & Cadeau & Corde & Ficelle \\
\hline Banane & Cadenas & Cornet & File \\
\hline Banc & Cadre & Cornichon & Filet \\
\hline Banquette & Café & Costume & Flamme \\
\hline Bateau & Cage & Coton & Flaque \\
\hline Béquille & Caillou & Couloir & Flèche \\
\hline Berceau & Caisse & Cour & Fléchette \\
\hline Bergère & Camera & Couronne & Fleur \\
\hline Biberon & Camembert & Coussin & Fleuve \\
\hline
\end{tabular}




\begin{tabular}{|c|c|c|c|}
\hline Flocon & Képi & Monnaie & Pelote \\
\hline Foin & Kermesse & Montagne & Peluche \\
\hline Foire & Kiwi & Montre & Pendule \\
\hline Forêt & & Moto & Perle \\
\hline Fouet & Lac & Mouchoir & Pétale \\
\hline Four & Lait & Moule & Pétard \\
\hline Fourche & Laitage & Moulin & Pharmacie \\
\hline Fourchette & Lampe & Mousse & Photo \\
\hline Fraise & Lance & Mur & Pièce \\
\hline Framboise & Lavabo & Muraille & Piège \\
\hline Frite & Légume & & Pile \\
\hline Fromage & Lentille & Nappe & Piment \\
\hline Fruit & Lessive & Neige & Pin \\
\hline Fumée & Lilas & Nénuphar & Pince \\
\hline Fusée & Linge & Niche & Ping-pong \\
\hline \multirow[t]{2}{*}{ Fusil } & Lit & Nid & Pioche \\
\hline & Livre & Noix & Pipe \\
\hline Galette & Local & Noisette & Piscine \\
\hline Gant & Locomotive & Note & Piste \\
\hline Garage & Logis & Nouille & Plafond \\
\hline Gare & Loterie & Noyau & Planche \\
\hline Gâteau & Louche & Nuage & Plante \\
\hline Gilet & Loupe & Numéro & Plat \\
\hline Glace & Luge & & Plateau \\
\hline Gland & Lumière & Océan & Pluie \\
\hline Gomme & Lune & Euf & Plume \\
\hline Goutte & Lunette & Olive & Poignée \\
\hline Grain & & Orage & Poing \\
\hline Graine & Machine & Orange & Pointe \\
\hline Grillage & Maille & Oreiller & Poire \\
\hline Grotte & Maillot & & Poireau \\
\hline Gruyère & Malle & Paille & Pois \\
\hline Guidon & Mandarine & Pain & Poison \\
\hline \multirow[t]{2}{*}{ Guitare } & Manège & Palais & Poivre \\
\hline & Mare & Panier & Poivron \\
\hline Habitation & Marmite & Pantalon & Pomme \\
\hline Hache & Marteau & Pantin & Pont \\
\hline Hangar & Masque & Papier & Portail \\
\hline Haricot & Mèche & Paquet & Porte \\
\hline \multirow[t]{2}{*}{ Herbe } & Médaille & Parachute & Pot \\
\hline & Melon & Parasol & Potager \\
\hline Image & Menu & Parc & Potiron \\
\hline \multirow[t]{2}{*}{ Igloo } & Mer & Pâte & Poulie \\
\hline & Météo & Pâté & Poupée \\
\hline Jambon & Mètre & Patin & Prison \\
\hline Jeu & Métro & Patinoire & Prune \\
\hline Jouet & Meule & Patte & Puits \\
\hline Journal & Miel & Pastèque & Purée \\
\hline Judo & Miette & Pêche & Puzzle \\
\hline Jupe & Mine & Pédale & \\
\hline Jus & Miroir & Peigne & Quai \\
\hline
\end{tabular}




\begin{tabular}{|c|c|c|c|}
\hline Quille & $\begin{array}{l}\text { Table } \\
\text { Tableau }\end{array}$ & $\begin{array}{l}\text { Vigne } \\
\text { Village }\end{array}$ & Guerre \\
\hline Racine & Tabouret & Ville & Horreur \\
\hline Radis & Tâche & Violon & \\
\hline Rail & Taille-crayon & Vis & Idée \\
\hline Raisin & Tanière & Voiture & Indiscrétion \\
\hline Rame & Tapis & Volcan & Inondation \\
\hline Raquette & Tarte & Volet & \\
\hline Rasoir & Tartine & & Liquide \\
\hline Râteau & Tasse & Wagon & \\
\hline Ravioli & Téléphone & & Magie \\
\hline Règle & Télévision & Yaourt & Malheur \\
\hline Réveil & Tempête & & Manie \\
\hline Rideau & Tennis & $\underline{\text { Noms «abstraits" }}$ & Membre \\
\hline Rivière & Tente & Accident & Ménage \\
\hline $\operatorname{Riz}$ & Terre & Achat & Méprise \\
\hline Robinet & Tétine & Aide & Merveille \\
\hline Rocher & Thé & Appétit & Métal \\
\hline Rose & Tige & Articulation & Moisson \\
\hline Roti & Timbre & Assurance & Monde \\
\hline Roue & Tiroir & Aventure & Moyen \\
\hline Roulette & Tissu & & \\
\hline Route & Toboggan & Baignade & Naissance \\
\hline Ruche & Toile & Bataille & Nature \\
\hline Rue & Toit & Bêtise & Nouvelle \\
\hline & Toiture & & \\
\hline Sabot & Tomate & Calme & Odeur \\
\hline Sac & Tour & Choix & Odorat \\
\hline Salade & Tournevis & Colère & Ordre \\
\hline Saladier & Tournoi & Construction & Origine \\
\hline Salle & Tracteur & Conversation & Ouïe \\
\hline Sapin & Train & Cri & \\
\hline Saucisse & Trèfle & & Panache \\
\hline Saucisson & Tremblement & Danger & Parole \\
\hline Savon & Tromblon & Décollage & Pays \\
\hline Sel & Trompette & Demeure & Pelage \\
\hline Semoule & Trottoire & Documentaire & Plainte \\
\hline Sifflet & Trou & & Plongeon \\
\hline Sirop & Trousse & Elevage & Plastique \\
\hline Ski & Tuyau & Equilibre & Porcelaine \\
\hline Sol & & Espoir & Précipitation \\
\hline Soleil & Vague & Evènement & Profondeur \\
\hline Soupe & Valise & Expédition & Provenance \\
\hline Soupière & Vase & & Provision \\
\hline Source & Vélo & Famille & \\
\hline Sport & Velours & Folie & Race \\
\hline Statue & Vent & Frayeur & Région \\
\hline Stylo & Verre & & Rêve \\
\hline Sucette & Veste & Goût & Rôle \\
\hline Sucre & Vêtement & Goulée & Richesse \\
\hline & Viande & Grimace & \\
\hline
\end{tabular}




\begin{tabular}{|c|c|c|c|}
\hline Secousse & Colonne & Histoire & Quadrillage \\
\hline Secret & Comptine & Hiver & Quantité \\
\hline Semence & Conte & & Quartier \\
\hline Sens & Consigne & Infinitif & Question \\
\hline Sommeil & Copie & Instant & \\
\hline Souffre & Couleur & Intérieur & Rangée \\
\hline Spectacle & $\begin{array}{l}\text { Correction } \\
\text { Côté }\end{array}$ & Instrument & $\begin{array}{l}\text { Recette } \\
\text { Recréation }\end{array}$ \\
\hline Température & Croix & Jour & Rectangle \\
\hline Ton & & Journée & Rentrée \\
\hline Toucher & Date & & Résumé \\
\hline Tribu & Dé & Kilo & Résultat \\
\hline \multirow[t]{2}{*}{ Troupeau } & Début & & Retenue \\
\hline & Déjeuner & Lecture & \\
\hline Vérité & Départ & Lendemain & Semaine \\
\hline Vertige & Dessin & Lettre 1 et 2 & Signature \\
\hline Vie & Devinette & Ligne & Somme \\
\hline Visite & Devoir & Liste & Son \\
\hline Voisinage & Dictée & Longueur & Sortie \\
\hline \multirow[t]{2}{*}{ Vue } & Dictionnaire & & Soustraction \\
\hline & Direction & Majuscule & Suite \\
\hline Travail Scolaire & Dizaine & Marque & Syllabe \\
\hline$\overline{\text { Accent }}$ & Droite 1 et 2 & Matin & \\
\hline Accessoire & & Mémoire & Tableau \\
\hline Activité & Ecole & Mètre & Taille \\
\hline Addition & Ecriture & Milieu & Temps \\
\hline Affiche & Editeur & Modèle & Texte \\
\hline Anniversaire & Egalité & Morceau & Tiret \\
\hline Appel & Emplacement & Mot & Titre \\
\hline Après-midi & Endroit & Musique & Trait \\
\hline Ardoise & Entrée & & Travail \\
\hline Atelier & Episode & Nom & Triangle \\
\hline Auteur & Erreur & Nombre & Tranche \\
\hline \multirow[t]{2}{*}{ Automne } & Eté & & \\
\hline & Etiquette & Objet & Unité \\
\hline Bord & Exercice & Opération & \\
\hline Brouillon & Extérieur & & Vacances \\
\hline \multirow[t]{2}{*}{ Bulle } & Evaluation & Page & Verbe \\
\hline & Eveil & Parcours & \\
\hline Cache & & Part & Locutions verbales \\
\hline Case & Fin & Partie & (se mettre à l')Abri \\
\hline Cahier & Fois & Pastille & (être en) Âge de \\
\hline Calcul & & Peinture & (avoir de l')Argent \\
\hline Calendrier & Gabarit & Période & (faire) Attention \\
\hline Carré & Gauche & Phrase & \\
\hline Catalogue & Goûter & Poésie & (avoir) Besoin de \\
\hline Chose & Gramme & Poids & \\
\hline Centimètre & Groupe & Point & (faire un) Caprice \\
\hline Classe & & Prénom & (avoir la) Chance de \\
\hline Code & Hauteur & Printemps & (avoir de la) Chance \\
\hline Collection & Heure & & (être en) Chasse de \\
\hline
\end{tabular}


Etre le comble de Tenir compte de

(faire) Demi-tour (avoir le) Droit de (avoir des) Doutes

(prendre son) Élan (avoir l')Énergie de (avoir) Envie de (prendre son) Envol (faire un) Essai

(avoir) Faim

(prendre la) Fuite

(avoir) Horreur de

(avoir l')Impression de (avoir l')Intention de (se mettre à la) Place de (avoir de la) Peine (avoir, faire) Peur (partir à la) Recherche de (se mettre en) Route (aller au) Secours de

Chair

Menton

(avoir) Soif

Cheveu

Moustache

(avoir de la) Suite dans les idées

Cheville

Museau

(avoir, prendre le) Temps de

Locutions adverbiales

À volonté

À son tour

De justesse

De la même façon

En commun

En réalité

En retard

En sursaut

Sans réplique

Parties du corps

(bumain ou animal)

Aile

Babine

Barbe

Bec

Bouche
Cil

Cocon Narine

Cœur Nez

Corps

Cou

Ongle

Coude

Oreille

os

Défense

Dent Pied

Doigt Plancton

Dos Poil

Pouce

Epaule

Griffe

Queue

Tête

Hanche

Ventre

Jambe

Visage
Langue

Main 